\title{
Gasless laparoscopy revisited in the SARS-CoV-2 pandemic
}

\author{
Ahmed Ali Abdelbaset ${ }^{1,2} \cdot$ Amulya K. Saxena $^{1}$ (i) \\ Received: 17 April 2020 / Revised: 12 May 2020 / Accepted: 13 May 2020 / Published online: 28 May 2020 \\ (c) Springer Nature Singapore Pte Ltd 2020
}

\begin{abstract}
Reluctance towards general acceptance of gasless laparoscopic surgery has been mainly due to reservations on whether these techniques would create a greater extent of pain and surgical stress at the targeted site of abdominal wall lifting, and also whether the operating space achieved would be sufficient for the scope to be maneuvered effectively and safely in the abdominal cavity. With the widespread mortality in patients infected by SARS-CoV-2, the most relevant gasless endoscopic techniques have been revisited to explore options in performing endoscopic procedures, especially in children with more safety by reducing aerosolized viral contamination.
\end{abstract}

Keywords Gasless · Laparoscopy · COVID-19 · SARS-CoV-2 $\cdot$ Techniques $\cdot$ Pediatrics

\section{Introduction}

The severe acute respiratory syndrome coronavirus 2 (SARS-CoV-2) also known as Coronavirus disease 2019 (COVID-19) outbreak started in December 2019 in Wuhan, China and has been declared a pandemic by the World Health Organization (WHO). This has impacted endoscopic surgery as concerns have been raised with regards to transmission of the virus by surgical smoke generated during these procedures and contamination during their release of pneumoperitoneum (PP) [1]. During laparoscopy, the PP is maintained under pressure within the abdominal cavity by insufflated carbon dioxide $\left(\mathrm{CO}_{2}\right)$. Smoke generated by energy-generating devices during such procedures is released into the operating room environment through positive intra-abdominal pressure. There is clear evidence that laparoscopy can lead to aerosolization of viruses [2]. In the past, skepticism towards general acceptance of gasless laparoscopic surgery was mainly due to reservations on greater extent of pain at the targeted site of abdominal wall

Amulya K. Saxena

amulya.saxena@nhs.net

1 Department of Pediatric Surgery, Chelsea Children's Hospital, Chelsea and Westminster Hospital NHS Fdn

Trust, Imperial College London, 369 Fulham Road, London SW10 9NH, UK

2 Pediatric Surgery Unit, Sohag Faculty of Medicine, Sohag University Hospital, Sohag, Egypt lifting, and also whether sufficient operating space could be achieved for the scope to be maneuvered effectively and safely in the abdominal cavity [3]. Despite these concerns, with the widespread mortality in patients infected by SARS$\mathrm{CoV}-2$, gasless endoscopic techniques have to be revisited to provide options in performing endoscopic procedures with more safety by reducing aerosolized viral contamination. A non-systematic review of the literature was conducted from Pubmed database to retrieve the most 5 most relevant scientific publications that have been identified and elaborated in this communication.

\section{Balloon laparoscopy [4]}

The balloon laparoscopy system consists of a stabilizing metal sleeve with a transparent balloon attached at one end, with the other end having a sealing closure device. After gaining access to the abdominal cavity, the balloon system is introduced with the aid of a guide rod. The guide rod is retracted and the scope is the introduced into the balloon system after which the system is sealed by the closure device. Once in position, the balloon is inflated with air. As there is no lift to the abdominal wall, this system is restricted to exploration of organs and obtaining biopsies, but not suitable for interventions. 


\section{Gasless laparoendoscopic single site (LESS) surgery} [5]

For these surgical procedures performed in the pediatric age group of 6-19 year olds, an Alexis wound retractor (Applied Medical, Rancho Santa Margarita, CA) is utilized as a single-access working port with placement through a $2.5-\mathrm{cm}$ midline umbilical skin incision. Surgical procedures can then be performed with conventional laparoscopic instruments under vision with a rigid scope. Though gasless LESS procedures offer easy anatomical access and easier retrieval of the bulky masses (such as ovarian dermoid cyst), a significant disadvantage is the difficult intracorporeal triangulation of surgical instruments. Furthermore, sometimes establishing the sufficient working space could be difficult due to poor expansion of the abdominal cavity.

\section{Kirschner wire lift}

(i) Abdominal wall wire lift [6]: In this technique, a $10-\mathrm{mm}$ trocar is employed to puncture the right superior border of the umbilicus through which a scope is positioned. The patient is then positioned in a Trendelenburg's position and a Kirschner wire (1.2-1.5 in diameter) is adopted to cross subcutaneously about $4 \mathrm{~cm}$ from the upper middle part of medioventral line of the lower abdomen in a horizontal direction, after which the wire is pierced out from the skin. Lifting devices are then used to lift abdominal wall with the desired lifting height adjusted by lifting chain.

(ii) Costal arch wire lift [7]: This method is employed for left upper quadrant access (splenectomy) with the patient placed in the right semilateral position. A $12-\mathrm{mm}$ port is inserted near the umbilicus or left upper quadrant, after which the left costal arch and left upper quadrant are lifted using a subcutaneous Kirschner wire attached to an abdominal wall-lift system. After this mechanical lift, surgical procedures can performed on the spleen; however, the authors state that despite this technique of gasless laparoscopic splenectomy being feasible, there are disadvantages particularly with regards to the restricted operative working space in some patients.

\section{Abdominal retractor lift [8]}

In this technique, two towel clips are used to hold the lower end of the umbilicus up and an incision of $3 \mathrm{~cm}$ is made large enough to introduce a finger. The abdominal lift device is used to lift the anterior abdominal wall under vision using a carefully inserted retractor. The lift apparatus is designed such that it lifts the abdominal wall by raising the umbilicus $10 \mathrm{~cm}$ and, hence, providing sufficient space to operate. Combinations of open and laparoscopic instruments can be used for the surgical procedure. Since no gas is introduced, larger instruments could also be utilized.

\section{Area lift [9]}

In this lift technique, subcutaneous 10-20-mm-thick and 15-40-cm-long wires (depending on the age of the patient) are placed subcutaneously through a stab wound. Each wire is held by thick sutures to one of a pair of metal plates with multiple holes for "hanger lifting". The metal plates are maintained by clamp hooks attached to an arch that is placed across the body of the patient with the two ends fixed at both sides of the operating table. Simultaneous lifting of a pair of wires produces a wide roof-shaped intra-abdominal space sufficiently wide for safe placement of 3-5 valveless ports for laparoscopic procedures. This technique is reported in pediatric surgical patients with the youngest patient operated being 8 days old. This gasless laparoscopic surgery using subcutaneous wiring has also been reported to be more readily applicable to children than to adults who have abundant subcutaneous adipose tissue.

\section{Discussion}

Although five broadly characterized techniques have been identified in the surgical implementation of gasless laparoscopy, multiple modification of these systems is available. Whereas the balloon laparoscopy is specifically tailored for diagnostic purposes, the other four techniques LESS, Kirschner wire lifts, abdominal retractor lift and area lift enable the possibility of interventions. Most of these techniques have been reported in adults and none of them have been systematically implemented or investigated in a larger pediatric surgical group of patients with regards to complications and technical difficulties in application. Post-operative complications using these techniques are reported to be related to the abdominal incision and are similar to the complications of open surgery: parietal hematoma, infection at the incision site and dehiscence, but with increased pain at the site of abdominal lifts [10].

Despite this, proponents of gasless laparoscopy have focused on the advantages over CO2-PP which was related to cardiopulmonary compromise as a potentially life-threatening event and risk factors involving alterations in central venous pressure, various respiratory and endocrinologic parameters, hypothermia, and gas embolism;[3]; however, during the present COVID-19 pandemic efficacy of gasless 
laparoscopy options even in pediatrics will have to be reexplored now more in the interest of public safety with regards to minimizing SARS-CoV-2 transmission from patients to operating room staff.

\section{References}

1. SAGES.org [Internet] SAGES and EAES Recommendations Regarding Surgical Response to COVID-19 Crisis-SAGES (2020) https://www.sages.org/recommendations-surgical-response-covid -19/?fbclid=IwAR2M139pV3bwWp9XYB2cfWyYgrnxbks0W 3GZ_sZ0GrGXVucZQULXqOf7Jfk.

2. Kwak HD, Kim SH, Seo YS et al (2016) Detecting hepatitis B virus in surgical smoke emitted during laparoscopic surgery. Occup Environ Med 73:857-863

3. Ren H, Tong Y, Ding X-B et al (2014) Abdominal wall-lifting versus $\mathrm{CO} 2$ pneumoperitoneum in laparoscopy: a review and meta-analysis. Int J Clin Exp Med 7:1558-1568

4. Volland M, Lienert M, Horstmann O (2010) Gasless balloon laparoscopy. Surg Endosc 24:1566-1572
5. Takeda A, Imoto S, Nakamura H (2014) Management of pediatric and adolescent adnexal masses by gasless laparoendoscopic single-site surgery. Eur J Obstet Gynecol Reprod Biol 181:66-71

6. Li SH, Deng J, Huang FT, Gan XW, Cao YG (2014) Impact of gasless laparoscopy on circulation, respiration, stress response, and other complications in gynecological geriatrics. Int J Clin Exp Med 7:2877-2882

7. Hyodo M, Sata N, Koizumi M et al (2012) Laparoscopic splenectomy using pneumoperitoneum or gasless abdominal wall lifting: a 15-year single institution experience. Asian J Endosc Surg 5:63-68

8. Kruschinski D (2007) Atlas of lift-laparoscopy: the new concept of gasless laparoscopy, 1st edn. CRC Press, London

9. Yokomori K, Terawaki K, Kamii Y et al (1998) A new technique applicable to pediatric laparoscopic surgery: abdominal wall "area lifting" with subcutaneous wiring. J Pediatric Sure 33:1589-1592

10. Moga MA, Arvatescu CA, Pratilas GC et al (2015) The role of gasless laparoscopy in differential diagnosis of acute abdomen. Hippokratia 19:69-72 